\title{
Numerical Simulation of Leslie-Gower Predator-Prey Model with Stage-Structure on Predator
}

\author{
Rima Anissa Pratiwi, Agus Suryanto*, Trisilowati \\ Departemant of Mathematics, Faculty of Mathematics and Natural Sciences, University of Brawijaya, Malang, Indonesia
}

\begin{abstract}
In this paper, we introduce Leslie-Gower predator-prey model with a stage-structure population on the predator. This model consists of two populations, that are prey and predator populations. Here, we divide predator into two stages. Thus, we have three classes of population in this model that are prey, juvenile predator, and mature predator. The focus of this paper is to know the interaction between the population that is affected by stage-structure in predator population in the model and to study numerically the effects of stage-structure in predator population on the interaction of prey and predator. It is found that the trantition rate from juvenile to mature predator is a very important parameter which may determine the long-term behavior of both prey and predator.
\end{abstract}

Keywords: Leslie-Gower model, predator-prey model, stage-structure.

\section{INTRODUCTION}

Ecology is one branch of science from biology that is still frequently studied. One of the things studied in ecological problems is the interaction between organisms. The interaction between organisms cannot be separated from the problem of predation. Predation can be modeled into a mathematical model called a predatorprey model. By solving the model, we can predict the population behavior of both prey and predator in the future. One of the famous preypredator models is the Leslie-Gower model. Leslie-Gower [1] introduced a predator-prey model that assumes that predator grows logistically with its carrying capacity comparable to the number of prey populations.

Many studies have been produced using Leslie-Gower model. Aziz-Alaoui and Okiye [2] added environmental protection parameter to the Leslie-Gower model for the survival of predator. In this model, they use Holling Type-II functional response which states that predation depends only on the number of prey populations alone. In 2014, Yu [3] assumed that predation depends not only on prey population but also on predator due to interference between predator during predation. So he uses the BeddingtonDeAngelis functional response on this model. In 2016, Indrajaya [4] considered that the Allee effect is one of the factors that influence the interactions between prey and predator.

\footnotetext{
* Correspondence address:

Agus Suryanto

Email : suryanto@ub.ac.id

Address : Dept. Mathematics, Faculty of Mathematics and Natural Sciences, University of Brawijaya, Veteran Malang, 65145.
}

Leslie-Gower model has been studied by many researchers, but the age of the population is often be ignored. But, many cases in life consider that the population dynamics depend on the stage-structure, e.g. juvenile and mature population. This case is commonly encountered in mammals. In 1990, Aiello and Freedman [5] studied the model of one species undergoing two stage. This model assumes that the average age of the mature population is expressed as a delay time constant, this implies the late birth of juvenile population and reduced juvenile population that turn into mature population. In 2006, Cui and Yasuhiro [6] introduced predatorprey models with periodic functions with age structure on prey population. Then Cai and Song [7] studied predator-prey models whose predator population is divided into two stages.

In this paper, we modify Yu [3] model's and construct a Leslie Gower predator-prey model with stage-structure population on the predator, because the age of population can influence the evolution of the population. After that, we investigate the behavior of the model numerically and we will compare the behavior of model with or without the stage-structure on predator population.

\section{MATERIAL AND METHOD \\ Construction Model}

In this section, Leslie-Gower predator-prey model in $\mathrm{Yu}$ [3] is modified into Leslie-Gower predator-prey model with stage-structure on the predator. We modify Yu's predator-prey model [3] into a predator-prey model of Leslie-Gower with stage-structure on predator by adding the assumption that predator can be divided into two 
stages, that are juvenile predator and mature predator.

\section{Determining Equilibrium Points}

The equilibrium point is the solution of a model that has a constant value, which can be determined by solving:

$$
\frac{d x}{d t}=\frac{d y_{1}}{d t}=\frac{d y_{2}}{d t}=0 .
$$

\section{Numerical Simulation}

Numerical simulations are performed to present the interaction of each population in the system (1) and (2). In this paper, several different transition rate values $(\beta)$ are used to determine the effect of stage-structure on the model. Simulations are done by solving the model using the 4th order Runga Kutta method [8].

\section{RESULT AND DISCUSSION}

Mathematical Model

Leslie-Gower's predator-prey model on [4] is expressed as follows

$$
\begin{aligned}
& \frac{d x}{d t}=r_{1} x\left(1-\frac{x}{k_{1}}\right)-\frac{a x y}{a+b x+c y^{x}} \\
& \frac{d y}{d t}=r_{2} y\left(1-\frac{y}{x+k_{2}}\right) .
\end{aligned}
$$

In this model, it is presumed that the prey grows following the logistic growth rate with the birth rate of the prey $\left(r_{1}\right)$ and carrying capacity $\left(k_{1}\right)$. Predator can grow logistically with birth rate $\left(r_{2}\right)$ and carrying capacity depending on the number of prey populations and environmental protection $\left(k_{2}\right)$ for the survival of the predatory population. Predator can attack prey populations with predation rates modeled by the BeddingtonDeAngelis functional rensponse. This functional rensponse assumes that predation not only depends on the prey population but also on the predator population.

In this paper, we add the assumption that predator experiences stage-structure, that are mature predator and juvenile predator. We add the assumption that the birth rate of mature predator is derived from a juvenile that turn into mature predator with rate denoted by $\beta$. While mature predator has a natural rate of death that is symbolized by $Y$.

We obtain Leslie-Gower predator-prey model with stage-structure on predator as

$$
\frac{d x}{d t}=r_{1} x(t)\left(1-\frac{x}{k_{1}}\right)-\frac{a x y_{2}}{a+b x+c y_{2}{ }^{b}}
$$

$$
\begin{aligned}
& \frac{d y_{1}}{d t}=\gamma_{2} y_{1}(t)\left(1-\frac{y_{1}}{x+k_{2}}\right)-\beta y_{1 x} \\
& \frac{d y_{2}}{d t}=\beta y_{1}-\gamma y_{2} x
\end{aligned}
$$

with $r_{1}, r_{2}, a, b, c, k_{1}, k_{22_{a}} \alpha, \beta, \gamma>0$

\section{Equilibrium Points}

Model (2) has four equilibrium points, namely

1. Trivial equilibrium point $E_{0}=(0,0,0)$,

2. Predator-free equilibrium point $E_{1}=\left(k_{1}, 0,0\right)$,

3. Survival of predator equilibrium point

$$
E_{2}=\left(0, k_{2}\left(1-\frac{\beta}{r_{2}}\right), \frac{\beta}{\gamma} k_{2}\left(1-\frac{\beta}{r_{2}}\right)\right) \text {, }
$$

4. Interior equilibrium point

$$
\begin{aligned}
& E_{3}\left(x^{*}, y_{1}{ }^{*}, y_{2}{ }^{*}\right) \quad \text { where } \\
& y_{1}^{*}=\left(1-\frac{\beta}{r_{2}}\right)\left(x^{*}+k_{2}\right), \\
& y_{2}^{*}=\frac{\beta}{y}\left(1-\frac{\beta}{r_{2}}\right)\left(x^{*}+k_{2}\right) \text { and } x^{*} \text { are }
\end{aligned}
$$

positive solution of quadratic equation

$$
\left(b \gamma r_{1} r_{2}+c \beta r_{1} r_{2}-c \beta^{2} r_{1}\right) x^{*}+
$$$$
\left(c \beta^{2} k_{1} r_{1}-c \beta k_{1} r_{1} r_{2}-\alpha \beta^{2} k_{1}+\right.
$$$$
\alpha \beta k_{1} r_{2}+c \beta k_{2} r_{1} r_{2}+\alpha \gamma r_{1} r_{2}-
$$$$
\left.c \beta^{2} k_{2} r_{1}-b \gamma k_{1} r_{1} r_{2}\right) x^{*}+
$$$$
c \beta^{2} k_{1} k_{2} r_{1}-a \gamma k_{1} r_{1} r_{2}-
$$$$
c \beta k_{1} k_{2} r_{1} r_{2}+\alpha \beta k_{1} k_{2} r_{2}-
$$$$
\alpha \beta^{2} k_{1} k_{2}=0 \text {. }
$$

\section{Numerical Simulation}

In this section, some numerical simulations are carried out to see the interaction between the population in model (1) and (2). Numerical simulations are performed using parameters in Table 1.

Table 1. Parameters Value

\begin{tabular}{lccccccccc}
\hline Parameters & $r_{1}$ & $r_{2}$ & $k_{1}$ & $k_{2}$ & $\alpha$ & $\gamma$ & $a$ & $b$ & $c$ \\
\hline Value & 1.6 & 1.5 & 1.72 & 1.2 & 1 & 0.05 & 0.7 & 1 & 0.1 \\
\hline
\end{tabular}

Using parameters as in Table 1, we obtain a solution for model (1) as plotted in Figure 1. We can see that in this case, the two populations can live together and stable toward a certain value.

Next we perform simulation using the same parameters as in Table 1 , but with varying the value of $\beta$ to observe the effect of stagestructure on the model. The result is shown in Figure 2. We get that for a relatively small $\beta$ 
value, for example $\beta=0.75$ or $\beta=1.25$ then the population of prey may be extinct, while the predator (juvenile and mature) will survive. If we set $\beta=1.48$, then the three population will live together towards a certain value. And when $\beta=1.75$ and $\beta=2$, then the prey population will survive while the predator (juvenile and mature) will go to extinction. This shows that the value of transition rate $\beta$ is very important in this model.
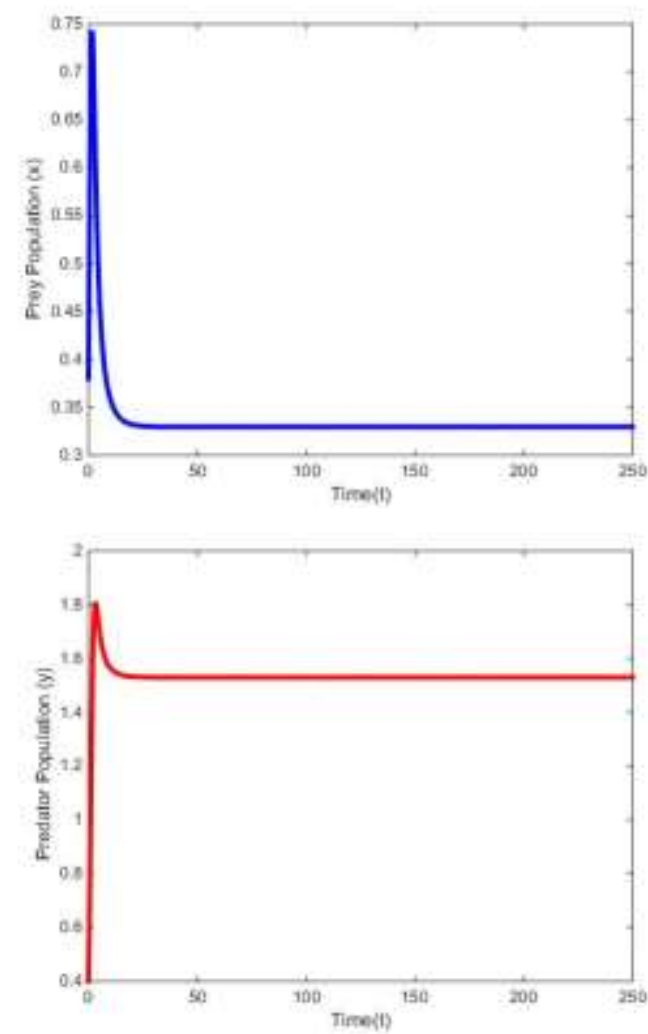

Figure 1. Numerical Solution of Model (1) for Prey and Predator Population.

To see the effect of $\beta$ on the behavior of the system, we perform simulation using $\beta \in[0.5,2]$ and it is found that for $\beta \in[0.5,1.2]$ shows the same behavior as $\beta=[1.2,1.4458]$. Therefore, we plot of the convergent solution was carried out for $\beta \in[1.2,2]$ to see better results. The plot of the convergent solution shown in Figure 3 . Notice in Table 1 that the birth of the predator is $r_{2}=1.5$. If $\beta>r_{2}$, then the predator (juvenile and mature) will extinct, while the prey survive. This indicates that the solution converges to the equilibrium point $E_{1}$.

While if $\beta<r_{2}$, then the predator will not extinct, and the prey may be extinct if the $\beta$ are relatively small. Such behavior indicates that the solution converges to the equilibrium point $E_{2}$. However, if $\beta$ are large enough but $\beta<r_{2}$, then all three populations can live together, indicating that the solution converges to the equilibrium point $E_{a}$.
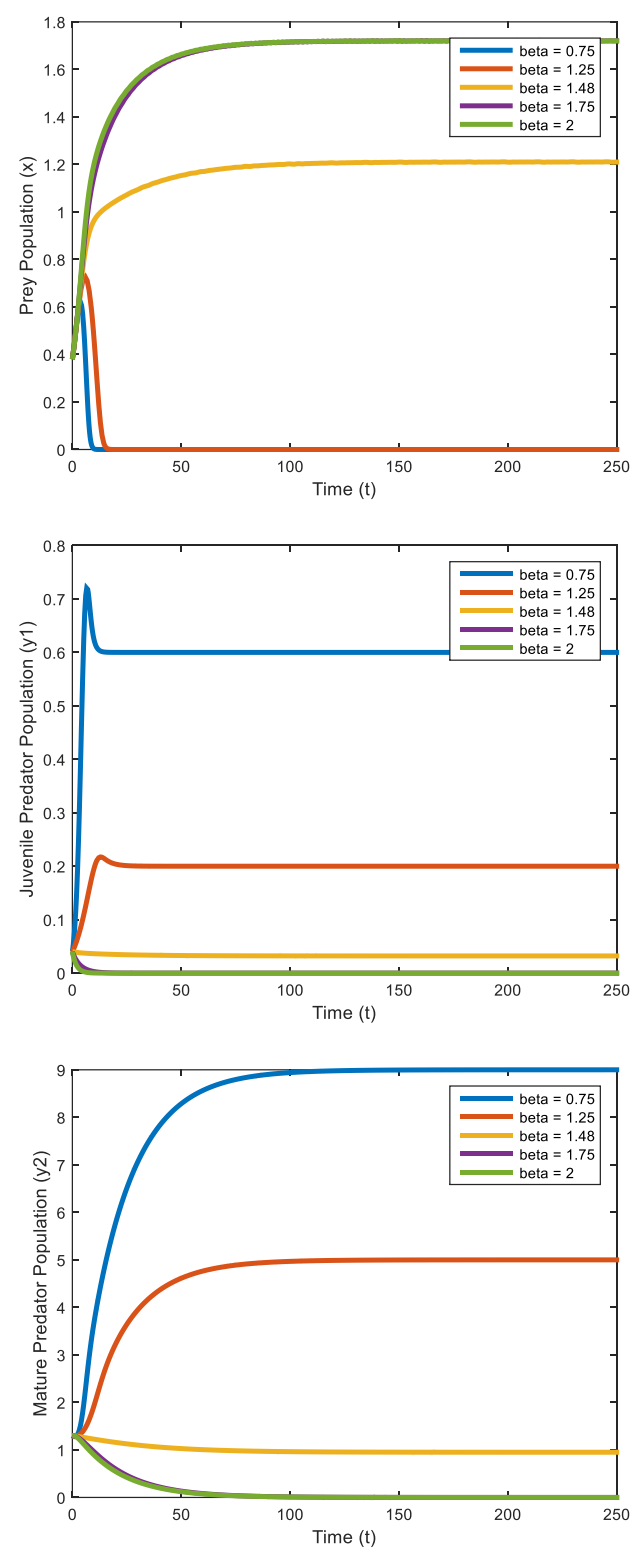

Figure 2. Numerical Solution of Model (2) for Prey, Juvenile Predator, and Mature Predator Population.

We can see from Figure 3 , there is an interval in $\beta$ value where the solution does not converge to any equilibrium point. In more detail, it is obtained numerically that if $0.5<\beta<1.4458$ then the solution will converge to $E_{2}$, if $1.4546<\beta<1.4955$ then the solution will converge to $E_{a}$, and if $1.5081<\beta<2$ then the solution will converge to $E_{1}$. If 
$1.4458<\beta<1.4546$ or $1.4955<\beta<1.5081$, then the solution is not convergent. For example, if we take $\beta=1.45$, the solution of model to (2) shown in Figure 4. It appears that the three populations live together with the periodically changing population.
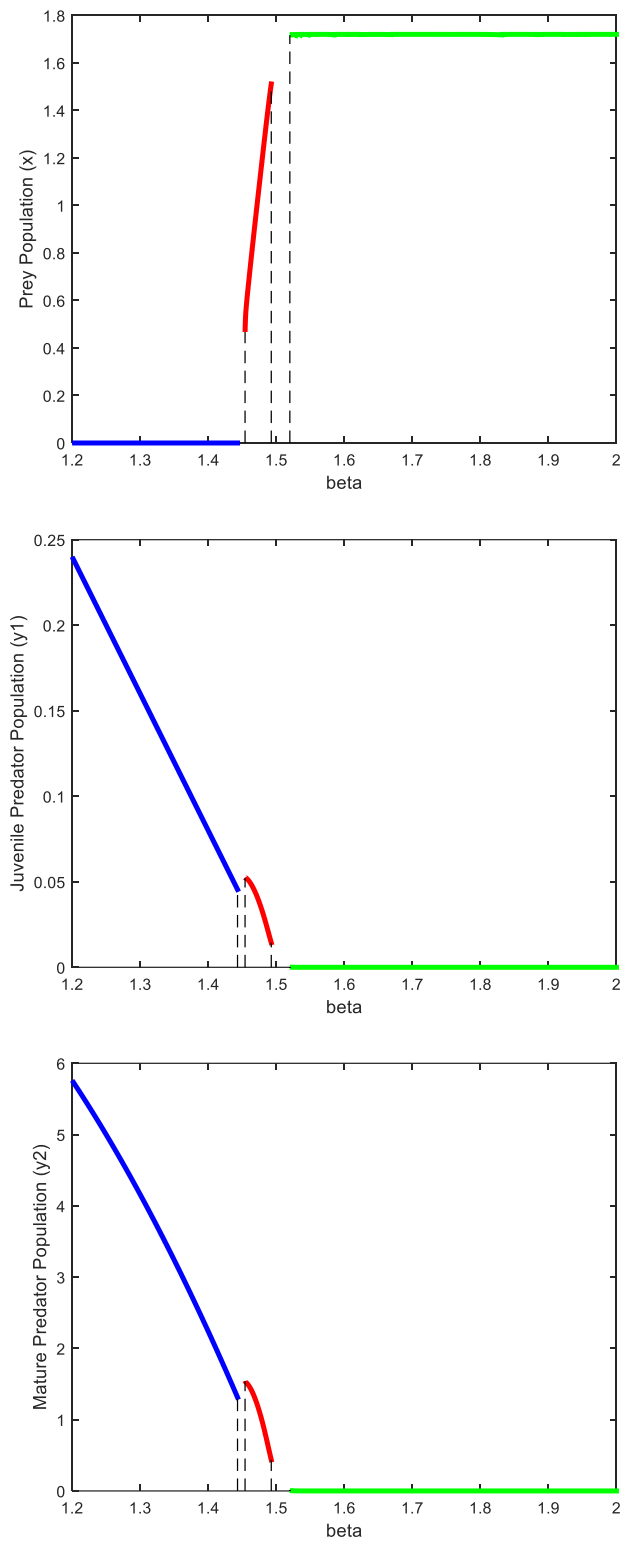

Figure 3. Convergent Solution of Model (2).

In mathematics, this condition indicates the presence of Hopf bifurcation. That is, there is a stability change in the model (2) because of the change in a parameter. In this case, the bifurcation parameter is $\beta$.

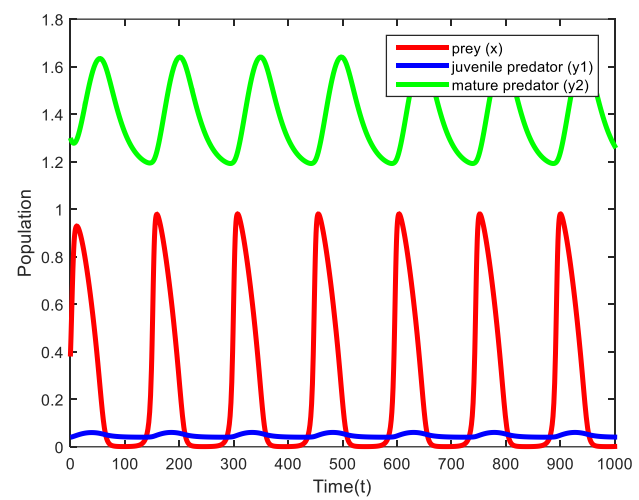

Figure 5. Solution of model (2) with $\beta=1.45$

\section{CONCLUSION}

In this paper, we proposed Leslie Gower predator-prey model with stage-structured on predator using the Beddington-DeAngelis functional response. We obtained the solution of the model numerically shown in Figure 2. Then we compare the solution behavior that occurs in the model with the solution behavior in the Leslie-Gower predator-prey model without stagestructure. It is shown that we have four equilibrium from model (2), that are $E_{0}, E_{1}, E_{2}$, and $E_{2}$. We get that if we set $\beta>r_{2}$, then the predator (juvenile and mature) will extinct, while the prey survive or the solution converges to $E_{1}$. But if we set $\beta<r_{2}$, then the predator will not extinct, and the prey may be extinct if the $\beta$ are relatively small or the solution converges to the equilibrium point $E_{2}$. However, if $\beta$ are large enough but $\beta<r_{2}$, then all three populations can live together which indicate that the solution converges to the equilibrium point $E_{3}$.

And if we compare the solution of model (2) with the solution behavior in the Leslie-Gower predator-prey model without stage-structure, it can be seen that with stage-structure making the behavior of the model more variable depending on the value of $\beta$ and it makes $\beta$ is very important parameter which may determined the long-term behavior of both prey and predator.

\section{REFERENCES}

[1] Leslie, P. H. 1958. A stochastic model for studying the properties of certain biological systems by numerical methods. Biometrika. 45(1-2).16-31.

[2] Aziz-Alaoui, M.A., M.D. Okiye. 2003. Boundedness and global stability for a predator-prey model with modified LeslieGower and Holling-type II schemes. Appl. Math. Lett. 16. 1069-1075. 
[3] Yu, S. 2014. Global stability of modified Leslie-Gower model with BeddingtonDeAngelis functional response. Advance in Difference Equation. $84 . \quad$ DOI: 10.1186/1687-1847-2014-84

[4] Indrajaya, D., A. Suryanto, A.R. Alghofari. 2016. Dynamics of modified Leslie-Gower predator-prey model with BeddingtonDeAngelis functional response and additive Allee effect. Int. J. Ecol. Dev. 31(3). 60-71.

[5] Aiello, W.G., H.I. Freedman. 1990. A timedelay model of single-species growth with stage structure. Math. Biosci. 101. 139-153.

[6] Cui, J., T. Yasuhiro. 2006. A predator-prey system with a stage structure for the prey. Math. Comput. Model. 44. 1126-1132.

[7] Cai, L., X. Song. 2007. Permanence and stability of a predator-prey system with stage structure for predator. J. Comput. Appl. Math. 201. 356-366.

[8] Suryanto, A. 2017. Metode numerik untuk persamaan diferensial biasa dan aplikasinya dengan MATLAB. State University of Malang. Malang. 